\title{
Nonlinear Steam Valve Adaptive Controller Design for the Power Systems
}

\author{
Nan Jiang, Xiangyong Chen, Ting Liu, Bin Liu, Yuanwei Jing \\ Faculty of Information Science and Engineering, Northeastern University, Shenyang, China \\ E-mail: jiangnan@ise.neu.edu.cn \\ Received October 21, 2010; revised December 9, 2010; accepted January 10, 2011
}

\begin{abstract}
Considering generator rotor and valve by external disturbances for turbine regulating system, the nonlinear large disturbance attenuation controller and parameter updating law of turbine speed governor system are designed using backstepping method. The controller not only considers transmission line parameter uncertainty, and has attenuated the influences of large external disturbances on system output. The nonlinear controller does not have the sensitivity to the influences of external disturbances, but also has strong robustness for system parameters variation, which is because of the transmission line uncertainty being considered in internal disturbances. The simulation results show that the control effect of the large disturbance attenuation controller more advantages by comparing with the control performance of conventional nonlinear robust controller.
\end{abstract}

Keywords: Power Systems, Steam Valve Control, Large Disturbance Attenuation, Backstepping, Adaptive

\section{Introduction}

With the development of large interconnected power systems and the use of kinds of new equipment, the size and complexity of power systems is increased, and it is inevitable that there have the influences of disturbances on the operation of power system. Meanwhile, owing to the inaccuracy of the model, the error of the parameters of the control object designed and the error of the measurement components of the controller also form the generalized disturbance of power system [1]. In order to improve the transient stability level of power system, and prevent an electric power system losing synchronism under a large sudden fault by closing the turbine regulating valve quickly to reduce the output of prime mover instantaneously, it is essential to design the strong robustness nonlinear steam valve controller to improve its reliability.

In recently years, various advanced nonlinear control technologies have been applied to excitation and steam valve controllers of power systems. Through careful investigation it is easy to see that most of these are based on some different control methods. However, these controllers suffer some flaws. Robust adaptive controller and nonlinear stabilizing controller of steam valve and excitation system design method are presented based on
Hamiltonian energy theory in [2,3], but it is difficult to solve the Hamilton-Jacobi-Issacs (HJI) inequality. In order to avoid solving the HJI inequality, $\mathrm{Lu}$ [1] designed the nonlinear L2 gain disturbance attenuation excitation controller based on recursive design method for the external disturbance attenuation problem. $\mathrm{Li}$ [4] developed a multivariable inverse nonlinear control scheme for strongly nonlinear reheat type turbo generator steam valves, which provides whole range control to improve the transient power system stability. In [5-7], the turbine governor and excitation controller design method using no linearization methods when system parameters are precisely knowable are proposed, but the robustness of the parameters and model was not considered. For a single-machine infinite -bus system, Wang [8] designed a nonlinear robust integrated controller and a parameter-update law is obtained based on adaptive backstepping methods and Lyapunov functions of the system. The controller also has strong robustness for system parameters variation because damping coefficient uncertainty has been considered. In order to prevent an electric power system losing synchronism under a large sudden fault and to achieve voltage regulation, Wang [9] applied the Riccati equation approach, together with the direct feedback linearization (DFL) technique to design robust nonlinear controllers for transient stability enhancement 
and voltage regulation of power systems under a symmetrical three-phase short circuit fault. Sun [10] designed the nonlinear adaptive controllers and parameter updating laws of the switch subsystems using the proposed modified adaptive backstepping method for a single- machine-infinite-bus system, the control input constraints are solved by introducing a switched mechanism. In [11-13], the nonlinear adaptive controllers and parameter updating laws for a single-machine-infinite- bus system with damping coefficient uncertainties in the turbine steam valve control also are proposed. However, the influences of the external unknown disturbances are not considered. Thus, how to effectively deal with the influences of the external unknown disturbances on system output for the origin system is valuable to be further studied.

Thus, in this paper we use the backstepping method to design the nonlinear disturbance attenuation controller and parameter updating law of turbine speed governor system for the uncertainty of transmission line parameters and the influences of large external disturbances on system output. In the design process, the influences of disturbances are considered while it does not use any linearization methods. Thus, the nonlinear controller does not have the sensitivity to the influences of external disturbances, but also keep the nonlinearities of systems. The simulation result demonstrates that the disturbance attenuation controller possesses superior performances by comparing with the control performance of conventional nonlinear robust controller. The design procedure based on the adaptive backstepping method is systematic and concise, it is believed that this method can be accepted with a considerable ease by engineers.

\section{Model and Problem Statement}

Consider the following single-machine infinite-bus system with steam valve control and the configuration of whole system is shown in Figure 1.

Then the model of main steam-valve control system is expressed as follows [11]:

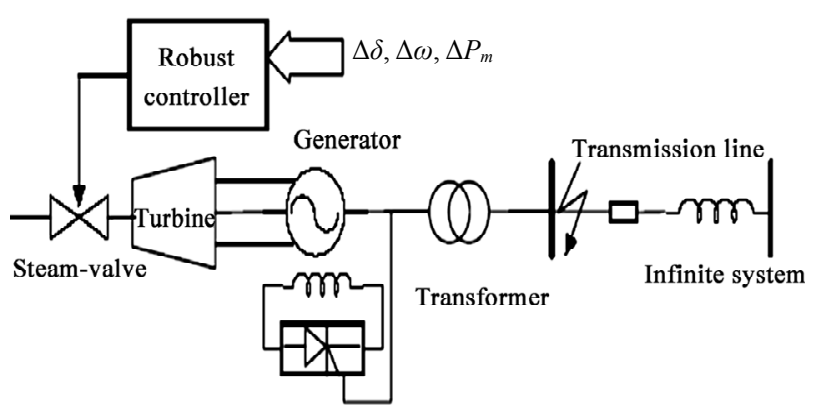

Figure 1. A single-machine infinite-bus system with the steam valve control.

$$
\left\{\begin{array}{l}
\dot{\delta}=\omega-\omega_{0} \\
\dot{\omega}=\frac{\omega_{0}}{H}\left(P_{H}+C_{M L} P_{m 0}-P_{\mathrm{e}}-P_{D}\right) \\
\dot{P}_{H}=-\frac{1}{T_{H \Sigma}}\left(P_{H}-C_{H} P_{m 0}+C_{H} u\right)
\end{array}\right.
$$

where $\delta$ is the rotor angle of generator. $\omega$ is the rotor speed of generator. $P_{m}, P_{e}, P_{D}, P_{H}$ are mechanical power of prime motor, active power of generator, damping power and mechanical power generated by highpressure cylinder, respectively. $H$ is the inertial coefficient of generator. $T_{H \Sigma}$ is the equivalent time constant of valve control, the value is about 0.4. $C_{H}$ is the distribution coefficient of high-pressure turbine, it is about 0.3. $C_{M L}$ is the distribution coefficient of middle and low-pressure turbine power, it is about 0.7. $P_{m 0}$ is the initial value of mechanical power, $P_{m 0}=P_{e 0} \cdot u$ is the steam-valve control. And we know that:

$$
P_{e}=\frac{E_{q}^{\prime} V_{S}}{X_{d \Sigma}^{\prime}} \sin \delta, \quad P_{D}=\frac{D}{\omega_{0}}\left(\omega-\omega_{0}\right)
$$

where $D$ and $E_{q}^{\prime}$ are damping coefficient and transient EMF of generator $q$ axis, respectively. $V_{S}$ is the infinite bus voltage. $X_{d \Sigma}^{\prime}$ is the equivalent reactance between generator and infinite bus system.

Next, we will research the design of the controller when the parameter $X_{d \Sigma}^{\prime}$ is unknown. For system (1), in order to transform it into the appropriate form in backstepping design, let $x_{1}=\delta-\delta_{0}, x_{2}=\omega-\omega_{0}$ and $x_{3}=P_{m}-P_{m 0}$, where $\delta_{0}, \omega_{0}, P_{m 0}$ are the initial value of corresponding variables, then system (1) can be rewritten as follows:

$$
\begin{aligned}
& \quad\left\{\begin{array}{l}
\dot{x}_{1}=x_{2} \\
\dot{x}_{2}=-\frac{D}{H} x_{2}+\frac{\omega_{0}}{H}\left(x_{3}+P_{m 0}+C_{M L} P_{m 0}-P_{e}\right) \\
\dot{x}_{3}=-\frac{1}{T_{H \Sigma}}\left(x_{3}+P_{m 0}-C_{H} P_{m 0}+C_{H} u\right)
\end{array}\right. \\
& k_{1}=-\frac{D}{H}, k_{2}=\frac{\omega_{0}}{H}, T=-\frac{1}{T_{H \Sigma}}, \\
& a_{0}=\frac{\omega_{0}}{H}\left(P_{m 0}+C_{M L} P_{m 0}\right), C=-\frac{C_{H}}{T_{H \Sigma}},
\end{aligned}
$$

and they are known constants. Further, let

$$
\theta=-\frac{\omega_{0} E_{q}^{\prime} V_{S}}{H X_{d \Sigma}^{\prime}}
$$

and they are unknown parameter because of $X_{d \Sigma}^{\prime}$. Finally, consider the exogenous disturbance $\varepsilon=\left[\begin{array}{ll}\varepsilon_{1} & \varepsilon_{2}\end{array}\right]^{T}$, where $\varepsilon_{1}$ and $\varepsilon_{2}$ is the disturbance on generator rotor and steam valve, respectively. Then system (3) is trans- 
formed into:

$$
\begin{aligned}
& \dot{x}_{1}=x_{2} \\
& \dot{x}_{2}=k_{1} x_{2}+k_{2} x_{3}+a_{0}+\theta k_{2} \sin \left(\delta_{0}+x_{1}\right)+\varepsilon_{1} \\
& \dot{x}_{3}=T\left(x_{3}-b_{0}\right)+C u+\varepsilon_{2} \\
& Z=\left(\begin{array}{c}
q_{1} x_{1} \\
q_{2} x_{2}
\end{array}\right)
\end{aligned}
$$

where $Z=\left[\begin{array}{ll}q_{1} x_{1} & q_{2} x_{2}\end{array}\right]^{T}$ is regulation output; $q_{1}$ and $q_{2}$ are the non-negative weight coefficient, which represent the weighted proportions between $x_{1}$ and $x_{2}$.

Now we can note that the system (7) is a control system with parametric uncertainties and unknown control gain, as well as unknown bounded exogenous disturbances. Our control objective is to keep the rotor stable and the system states are stabilized simultaneously.

\section{Design of Nonlinear Adaptive Controller with Large Disturbance Attenuation}

For the controlled object with the uncertain parameters and external disturbances, we can use the adaptive backstepping method to design the nonlinear robust controller of steam valve. We can design a suitable controller $u$ by constructing the storage function $V(x)$, and then the system satisfies:

$$
V(x(t))-V(x(0)) \leq \int_{0}^{T}\left(\gamma^{2}\|\varepsilon\|^{2}-\|z\|^{2}\right) d t
$$

where any $T>0$, the $L_{2}$ gain of system less than $\gamma$, and $\gamma$ is the disturbance attenuation constant.

Noticing the above-mentioned facts, the analysis method and design process of controller can be summarized as follows:

Step 1: For subsystem (4), let $x_{2}$ as the virtual control, and take stabilizing function $x_{2}^{*}=-c_{1} x_{1}, c_{1}>0$ is a design constant; suppose $e_{1}=x_{1}, e_{2}=x_{2}-x_{2}^{*}$, then subsystem (4) can be expressed as

$$
\dot{e}_{1}=e_{2}-c_{1} x_{1}
$$

Via taking the first Lyapunov function candidate as

$$
V_{1}=\frac{\sigma}{2} e_{1}^{2}
$$

where $\sigma>0$, then the time derivative of $V_{1}$ found is

$$
\dot{V}_{1}=\sigma e_{1} \dot{e}_{1}=\sigma e_{1}\left(e_{2}-c_{1} e_{1}\right)=\sigma e_{1} e_{2}-\sigma c_{1} e_{1}^{2}
$$

Step 2: By augmenting (9), the new Lyapunov function can be formed as

$$
V_{2}=V_{1}+\frac{1}{2} e_{2}^{2}
$$

Suppose that

$$
H_{1}=\dot{V}_{2}+\frac{1}{2}\left[\|Z\|^{2}-\gamma^{2}\left\|\varepsilon_{1}\right\|^{2}\right]
$$

and the performance index can be supposed as follows,

$$
J_{1}=\int_{0}^{\infty}\left(\|Z\|^{2}-\gamma^{2}\left\|\varepsilon_{1}\right\|^{2}\right) d t
$$

then one gets

$$
\begin{aligned}
H_{1}= & \dot{V}_{2}+\frac{1}{2}\left[\left(q_{1}^{2} x_{1}^{2}+q_{1}^{2} x_{2}^{2}\right)-\gamma^{2} \varepsilon_{1}^{2}\right] \\
= & \sigma e_{1} e_{2}-\sigma c_{1} e_{1}^{2} \\
& +e_{2}\left[k_{1} x_{2}+k_{2} x_{3}+a_{0}+\theta k_{2} \sin \left(\delta_{0}+x_{1}\right)+\varepsilon_{1}\right] \\
& +e_{2} c_{1} x_{2}+\frac{1}{2} q_{1}^{2} e_{1}^{2}+\frac{1}{2} q_{2}^{2}\left(e_{2}-c_{1} e_{1}\right)^{2}-\frac{1}{2} \gamma^{2} \varepsilon_{1}^{2}
\end{aligned}
$$

By deviating (12) about $\varepsilon_{1}$ and supposing the first derivation is equal to 0 , we can get

$$
e_{2}-\gamma^{2} \varepsilon_{1}=0
$$

then (13) can be obtained as follows,

$$
\varepsilon_{1}^{*}=\frac{1}{\gamma^{2}} e_{2}
$$

We continue to find the second derivation of (12), one gets

$$
\frac{\partial^{2} H_{1}}{\partial \varepsilon_{1}^{2}}=-\frac{\gamma^{2}}{2}<0
$$

Then there is the maximum value of $H_{1}$ about $\varepsilon_{1}$.

$$
\max H_{1}=\max \left\{\dot{V}_{2}+\frac{1}{2}\left[\|Z\|^{2}-\gamma^{2}\left\|\varepsilon_{1}\right\|^{2}\right]\right\}
$$

Now get the integral both sides of (14), we can get

$$
\begin{aligned}
& \max \int_{0}^{\infty} H_{1} d t \\
& =\max \left[\int_{0}^{\infty} \dot{V}_{2} d t+\frac{1}{2} \int_{0}^{\infty}\left(\|Z\|^{2}-\gamma^{2}\left\|\varepsilon_{1}\right\|^{2}\right) d t\right]
\end{aligned}
$$

Let $\bar{H}_{1}=\int_{0}^{\infty} H_{1} d t$, then

$$
\max \bar{H}_{1}=\max \left[\left(V_{2}(\infty)-V_{2}(0)\right)+\frac{J_{1}}{2}\right]
$$

Owing to $\frac{J_{1}}{2}=\bar{H}_{1}-\Delta V_{2}$, one gets

$$
\begin{aligned}
& \max \left(\frac{J_{1}}{2}\right)=\max \left(\bar{H}_{1}-\Delta V_{2}\right) \\
& \leq \max \left(\bar{H}_{1}\right)-\min \left(\Delta V_{2}\right)
\end{aligned}
$$

Remark 1: Suppose that the disturbance $\varepsilon_{1}$ makes $V_{2}$ reduce to 0 , i.e. $\min \left(\Delta V_{2}\right)=0$, that is, when the 
systems have the sufficiently large disturbances, $V_{2}$ is not reduced, and then, $\max \left(J_{1} / 2\right)$ is equivalent to $\max \left(\bar{H}_{1}\right)$, so $\varepsilon_{1}$ is really the worst disturbances for system.

Remark 2: Based on the equivalent analysis of $\max \left(\bar{H}_{1}\right)$ and $\max \left(J_{1} / 2\right), \varepsilon_{1}$ make $\bar{H}_{1}$ get the maximum value means that $\varepsilon_{1}$ makes $J_{1}$ also obtain maximum. While our ultimate goal is to make $J_{1}$ minimum, then explain $\varepsilon_{1}$ on the system's performance damage is the largest. If we substitute disturbances with such damage degree into systems, based on which we design the controller, and ensure the stability of the closed-loop system. Thus it shows that the system is not sensitivity for the influences of disturbances in theory.

Substituting (13) into (12), there is

$$
\begin{aligned}
H_{1}= & \sigma e_{1} e_{2}-\sigma c_{1} e_{1}^{2} \\
& +e_{2}\left[k_{1} x_{2}+k_{2} x_{3}+a_{0}+\theta k_{2} \sin \left(\delta_{0}+x_{1}\right)\right] \\
& +e_{2} c_{1} x_{2}+\frac{1}{2} \gamma^{2} e_{2}^{2}+\frac{1}{2} q_{1}^{2} e_{1}^{2}+\frac{1}{2} q_{2}^{2} e_{2}^{2} \\
& -q_{2}^{2} e_{2} c_{1} e_{1}+\frac{1}{2} q_{2}^{2} c_{1}^{2} e_{1}^{2}
\end{aligned}
$$

Suppose that

$$
\begin{aligned}
& \alpha=\sigma_{1} c_{1}-\frac{1}{2} q_{1}^{2}-\frac{1}{2} q_{2}^{2} c_{1}^{2}, \\
& h_{1}=\sigma+\frac{1}{2} q_{2}^{2} c_{1}+\frac{1}{2 \gamma^{2}} c_{1}, \\
& h_{2}=\frac{1}{2 \gamma^{2}}+c_{1}+\frac{1}{2} q_{2}^{2}+k_{1}
\end{aligned}
$$

Then

$H_{1}=-\alpha e_{1}^{2}+e_{2}\left[h_{1} x_{1}+h_{2} x_{2}+k_{2} x_{3}+a_{0}+\theta k_{2} \sin \left(\delta_{0}+x_{1}\right)\right]$

Consider $x_{3}$ as the virtual control, and define that $e_{3}=x_{3}-x_{3}^{*}$, then choosing the new virtual stabilization function as follows,

$$
x_{3}^{*}=\frac{1}{k_{2}}\left[-h_{1} x_{1}-h_{2} x_{2}-a_{0}-\theta k_{2} \sin \left(\delta_{0}+x_{1}\right)-c_{2} e_{2}\right]
$$

Let $n_{1}=k_{2} \sin \left(\delta_{0}+x_{1}\right)$, where $c_{2}$ is a positive number to be selected.

$$
x_{3}^{*}=\frac{1}{k_{2}}\left[-h_{1} x_{1}-h_{2} x_{2}-a_{0}-\bar{\theta} n_{1}-c_{2} e_{2}\right]
$$

We can obtain

$$
H_{1}=-\alpha e_{1}^{2}-c_{2} e_{2}^{2}+e_{2} \tilde{\theta} k_{2} \sin \left(\delta_{0}+x_{1}\right)
$$

where $\tilde{\theta}=\theta-\bar{\theta}, \bar{\theta}$ is the estimated value of $\theta$.

Step 3: By augmenting $V_{2}$, the new Lyapunov function can be formed as

$$
V_{3}=V_{2}+\frac{1}{2} e_{3}^{2}+\frac{1}{2 \rho} \tilde{\theta}^{2}
$$

where $\rho>0$ is the adaptive gain coefficient.

Define that

$$
H_{2}=\dot{V}_{3}+\frac{1}{2}\left[\|Z\|^{2}-\gamma^{2}\|\varepsilon\|^{2}\right]
$$

and the performance index as follows,

$$
J_{2}=\int_{0}^{\infty}\left(\|Z\|^{2}-\gamma^{2}\|\varepsilon\|^{2}\right) d t
$$

$$
\begin{aligned}
& \text { Because } \dot{V}_{3}=\dot{V}_{2}+e_{3} \dot{e}_{3}+\frac{1}{\rho} \tilde{\theta} \dot{\tilde{\theta}} \text {, and } \\
& \dot{x}_{3}^{*}=\frac{1}{k_{2}}\left[-h_{1} x_{1}-\left(h_{2}+c_{2}\right) \dot{x}_{2}-\dot{\bar{\theta}} n_{1}-\left(\bar{\theta} n_{2}+c_{1} c_{2}\right) x_{2}\right] \\
& =\frac{1}{k_{2}}\left\{-h_{1} x_{1}-\left(h_{2}+c_{2}\right)\left[k_{1} x_{2}+k_{2} x_{3}+a_{0}\right.\right. \\
& \left.\left.\quad+\theta k_{2} \sin \left(\delta_{0}+k_{2}\right)+\frac{1}{\gamma^{2}} e_{2}\right]-\dot{\bar{\theta}} n_{1}-\left(\bar{\theta} n_{2}+c_{1} c_{2}\right) x_{2}\right\}
\end{aligned}
$$

one gets

$$
\begin{aligned}
H_{2}= & \dot{V}_{2}+e_{3} \dot{e}_{3}+\frac{1}{\rho} \tilde{\theta} \dot{\tilde{\theta}}+\frac{1}{2}\left(q_{1}^{2} x_{1}^{2}+q_{2}^{2} x_{2}^{2}\right)-\frac{1}{2} \gamma^{2}\left(\varepsilon_{1}^{2}+\varepsilon_{2}^{2}\right) \\
= & -\alpha e_{1}^{2}-c_{2} e_{2}^{2}+e_{2} \tilde{\theta} k_{2} \sin \left(\delta_{0}+x_{1}\right)+\frac{1}{\rho} \tilde{\theta} \dot{\tilde{\theta}}-\frac{1}{2} \gamma^{2} \varepsilon_{2}^{2} \\
& +e_{3}\left[T\left(x_{3}-b_{0}\right)+c u+\varepsilon_{2}\right]+e_{3} \frac{1}{k_{2}}\left\{-h_{1} x_{2}-\left(h_{2}+c_{2}\right)\right. \\
& \times\left[k_{1} x_{2}+k_{2} x_{3}+a_{0}+\theta k_{2} \sin \left(\delta_{0}+x_{1}\right)+\frac{1}{\gamma^{2}} e_{2}\right] \\
& \left.-\dot{\bar{\theta}} n_{1}-\left(\bar{\theta} n_{2}+c_{1} c_{2}\right) x_{2}\right\}
\end{aligned}
$$

By deviating (15) about $\varepsilon_{2}$ and supposing the first derivation is equal to 0 , we can get

$$
\varepsilon_{2}^{*}=\frac{1}{\gamma^{2}} e_{3}
$$

To find the second derivation of (15), we can obtain

$$
\frac{\partial^{2} H_{2}}{\partial \varepsilon_{2}^{2}}=-\frac{\gamma^{2}}{2}<0,
$$

So there is the maximum value of $H_{2}$ about $\varepsilon_{2}$. Then, we know that $\varepsilon_{2}^{*}$ is the most disturbance of system to make a performance cost $J_{2}$ be the largest value.

Substituting (16) into (15), then

$$
\begin{aligned}
H_{2}= & -\alpha e_{1}^{2}-c_{2} e_{2}^{2}+e_{2} \tilde{\theta} k_{2} \sin \left(\delta_{0}+x_{1}\right) \\
& +\frac{1}{\rho} \tilde{\theta} \dot{\tilde{\theta}}-\frac{1}{2 \gamma^{2}} e_{3}+e_{3}\left[T\left(x_{3}-b_{0}\right)+c u+\frac{1}{\gamma^{2}} e_{3}\right]
\end{aligned}
$$




$$
\begin{aligned}
& +\frac{e_{3}}{k_{2}}\left\{-h_{1} x_{2}-\left(h_{2}+c_{2}\right)\left[k_{1} x_{2}+k_{2} x_{3}+a_{0}\right.\right. \\
& \left.\left.+\theta k_{2} \sin \left(\delta_{0}+x_{1}\right)+\frac{e_{2}}{\gamma^{2}}\right]-\dot{\bar{\theta}} n_{1}-\left(\bar{\theta} n_{2}+c_{1} c_{2}\right) x_{2}\right\}
\end{aligned}
$$

Choosing the feedback control as follows,

$$
\begin{aligned}
u= & \frac{1}{c}\left\{-T\left(x_{3}-b_{0}\right)-\frac{e_{3}}{\gamma^{2}}-\frac{1}{k_{2}}\left[-h_{1} x_{2}-\left(h_{2}+c_{2}\right)\right.\right. \\
& \times\left(k_{1} x_{2}+k_{2} x_{3}+a_{0}+\bar{\theta} n_{1}+\frac{e_{2}}{\gamma^{2}}\right)-\dot{\bar{\theta}} n_{1} \\
& \left.\left.-\left(\bar{\theta} n_{2}+c_{1} c_{2}\right) x_{2}\right] c_{3} e_{3}\right\}
\end{aligned}
$$

then,

$$
\begin{aligned}
H_{2}= & -\alpha e_{1}^{2}-c_{2} e_{2}^{2}+e_{2} \tilde{\theta} k_{2} \sin \left(\delta_{0}+x_{1}\right)+\frac{1}{\rho} \tilde{\theta} \dot{\tilde{\theta}} \\
& -\frac{e_{3}^{2}}{2 \gamma^{2}}-c_{3} e_{3}^{2}-\frac{e_{3}}{k_{2}}\left(h_{2}+c_{2}\right) \tilde{\theta} n_{1}
\end{aligned}
$$

In order to ensure that $H_{2} \leq 0$, suppose that

$$
\begin{aligned}
& e_{2} \tilde{\theta} k_{2} \sin \left(\delta_{0}+x_{1}\right)+\frac{1}{\rho} \tilde{\theta} \dot{\tilde{\theta}}-e_{3}\left(h_{2}+c_{2}\right) \tilde{\theta} \sin \left(\delta_{0}+x_{1}\right)=0 \\
& \tilde{\theta}\left(e_{2} n_{1}-\frac{1}{\rho} \dot{\bar{\theta}}-e_{3}\left(h_{2}+c_{2}\right) \sin \left(\delta_{0}+x_{1}\right)\right)=0
\end{aligned}
$$

Choosing the parameter update law

$$
\dot{\bar{\theta}}=\rho\left[e_{2} n_{1}-e_{3}\left(h_{2}+c_{2}\right) \sin \left(\delta_{0}+x_{1}\right)\right]
$$
then

By choosing the appropriate coefficient $\sigma$, so $\alpha \geq 0$,

$$
H_{2}=-\alpha e_{1}^{2}-c_{2} e_{2}^{2}-c_{3} e_{3}^{2} \leq 0
$$

Let $V(x)=2 V_{3}(x)$, one gets

$$
\dot{V}(x) \leq \gamma^{2}\|\varepsilon\|^{2}-\|z\|^{2}
$$

where $\varepsilon=\left[\begin{array}{ll}\varepsilon_{1} & \varepsilon_{2}\end{array}\right]^{T}$. When $x(0)=0$, we get the integral both sides to satisfy the inequality $(17)$, so there are the gain from disturbance to output.

Remark 3: when the system operates normally, then $0^{\circ}<\sigma<180^{\circ}$, and we can know that $\sin \left(\delta_{0}+x_{1}\right) \neq 0$, so the control is defined.

Now get the integral both sides of (17), the dissipation inequality can be obtained as follows,

$$
V(x(t))-V(x(0)) \leq \int_{0}^{T}\left(\gamma^{2}\|\varepsilon\|^{2}-\|z\|^{2}\right) d t
$$

so there is L2 gain from disturbance to the output of system. And when $\varepsilon=0$, the closed-loop error control system is asymptotic stable with the feedback control law $u$, then the closed-loop control system can be expressed as follows,

$$
\left\{\begin{array}{l}
\dot{e}_{1}=e_{2}-c_{1} e_{1} \\
\dot{e}_{2}=-c_{2} e_{2}-e_{1}+\frac{n_{1} \tilde{\theta}}{k_{2}^{2}} \\
\dot{e}_{3}=-c_{3} e_{3}+\frac{\left(c_{1}+k_{1}+c_{2}\right) n_{1}}{k_{2}^{2}} \tilde{\theta} \\
\dot{\bar{\theta}}=-\dot{\tilde{\theta}}=\gamma\left[\frac{e_{2}}{k_{2}}+\frac{\left(c_{1}+k_{1}+c_{2}\right) e_{3}}{k_{2}^{2}}\right] n_{1}
\end{array}\right.
$$

\section{Simulation Examples}

In this section, we firstly analyze the stability of the system by analyzing the state responses of system, which is generated by Simulink. Further, when the system parameters change under the fault that occurs, we observe the stability time of system with the influence of nonlinear steam valve adaptive robust controller, so we can analyze the stability of nonlinear steam valve adaptive robust controller of power system based on adaptive backstepping method, and the bounded ness of the state of system and whether to suppress the influences of large external disturbances on system output also can be analyzed.

\subsection{Simulation of General Nonlinear Robust Controller}

In this subsection, we can give the simulation analysis of general nonlinear robust controller, and the transient responding curves of the system output state and the controller output can be shown in Figure 2 and Figure 3.

The general nonlinear robust steam valve controller can be designed using the general backstepping method. As this robust controller, the time of the stability of system is longer and it will keep a slow convergence. Moreover, it will maintain the small oscillation stability within a certain range, and the control is ineffective.

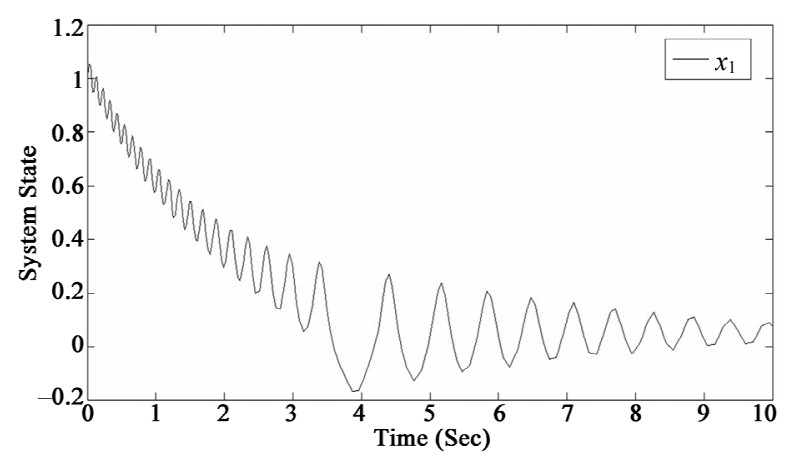

Figure 2. The transient responding curves of the system state $x_{1}$. 


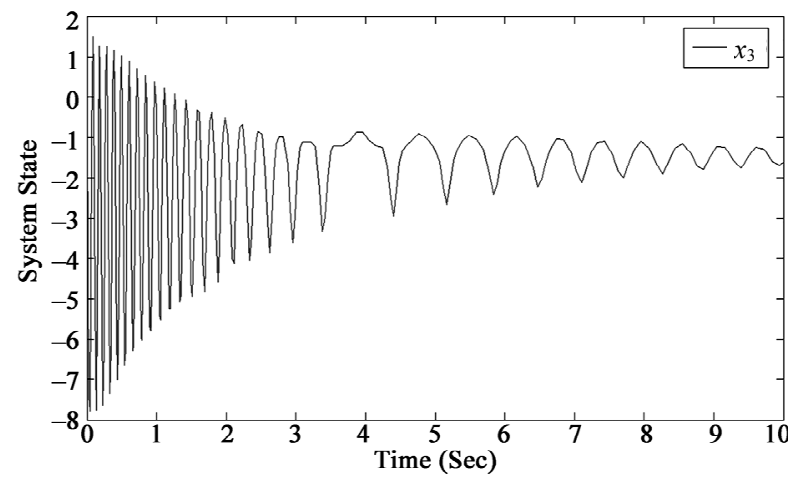

Figure 3. The transient responding curves of the system state $x_{3}$.

\subsection{Simulation of the Closed-Loop Error System}

According to the design results of foregoing section, we simulate system (18) using MATLAB. The simulation parameters can be supposed as follows: $D=5, H=8$, $c_{1}=2, c_{2}=2, c_{3}=2, \gamma=1, \rho=2, q_{1}=0.4$, $q_{2}=0.6, \sigma=1, \omega_{0}=314.159 \mathrm{rad} / \mathrm{s}, P_{m 0}=0.8 \mathrm{pu}$. And the responses of state can be obtained as Figure 4.

From the responding curves of the system states, we can see that the system have very good convergent performance and the closed-loop system goes into steady state quickly. When $t \rightarrow \infty$, then $e_{1} \rightarrow 0, e_{2} \rightarrow 0$, $e_{3} \rightarrow 0$. According to the definition of $x_{1}, x_{2}, x_{3}$ and $x_{2}{ }^{*}, x_{3}{ }^{*}, x_{1}, x_{2}$ and $x_{3}$ will be convergent to zero.
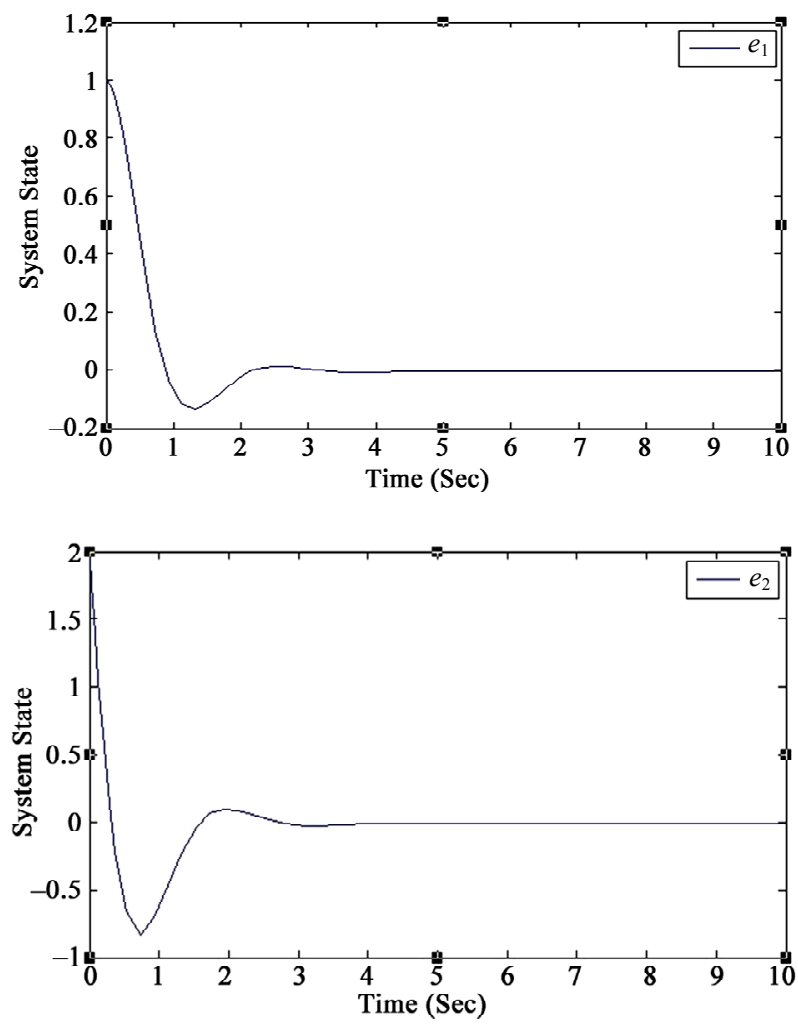
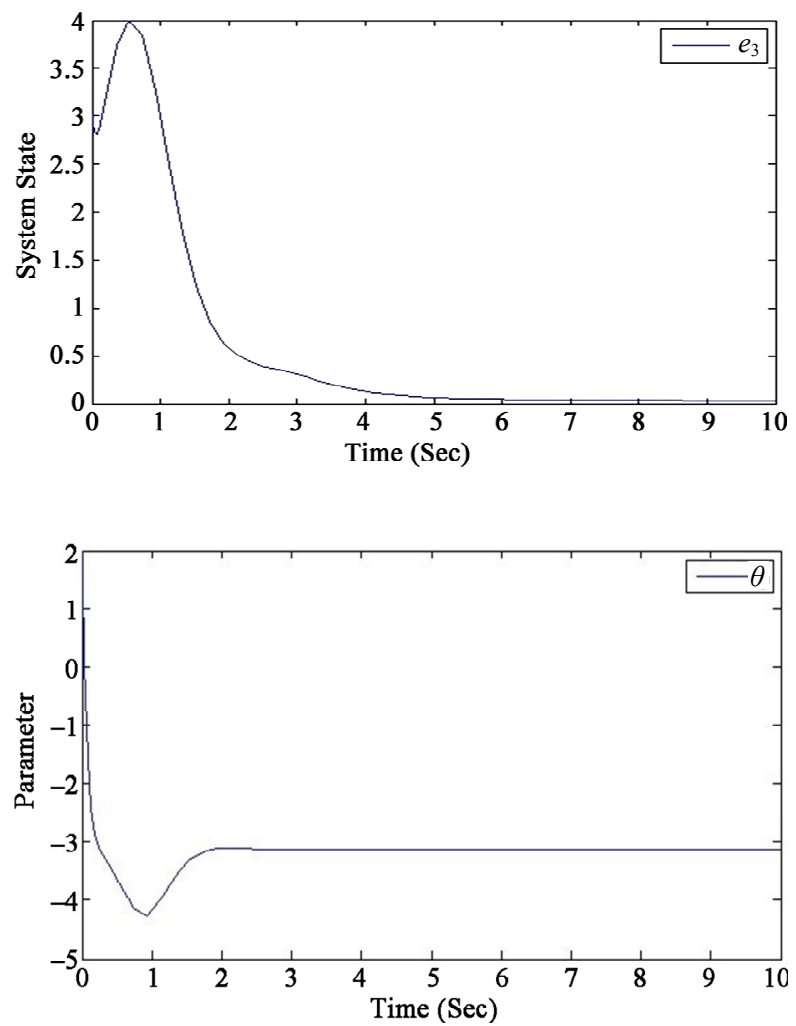

Figure 4. The state responding curves of the system.

\subsection{Simulation of Nonlinear Steam Valve Robust Controller}

In order to demonstrate superior performances of the controller designed by adaptive backstepping method, the external unknown disturbances of the generator rotor and steam valve are considered. And the transient responding curves of the controller can be shown in Figure 5.

From the responding curves of the system states, we can see that the system have very good convergent performance though being subjected to the influence of the maximum external disturbances, the speed that the closed-loop system goes into steady state is very quickly.

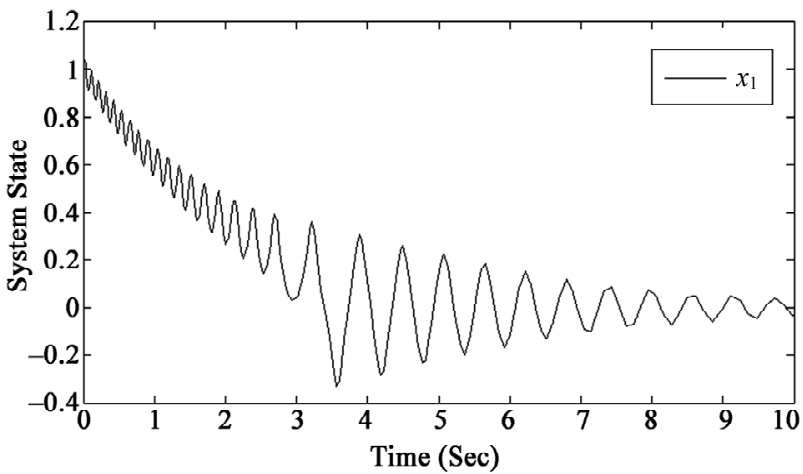




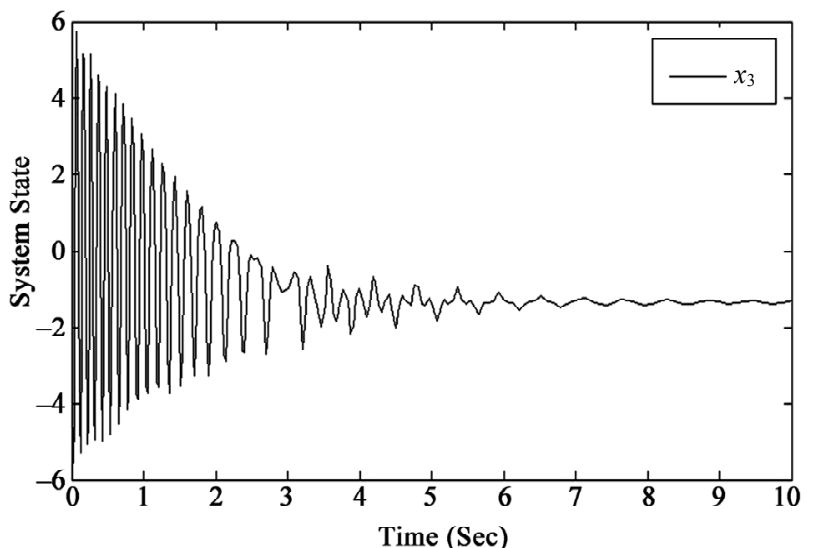

Figure 5. The response curve of the system state.

Thus all of these do verify the design process in this paper.

\section{Conclusion}

In this paper, we apply the nonlinear adaptive robust control scheme to turbine speed governor system. Nonlinear adaptive robust controller of steam-valve with the large external disturbances attenuation technique is designed by combining adaptive backstepping method. The essential nonlinear nature of the systems is entirely preserved because of no use of any kind of linearization on the original nonlinear system model. Owing to the fact that both internal and external disturbances in controller design are considered together, we compared the designed controller with the more conventional, not only the designed nonlinear controller does not have the sensitivity to the influences of external disturbances, but also has strong robustness for system parameters variation. The further simulations demonstrated the controller has superior adaptability and robustness.

\section{Acknowledgements}

This work is supported by the Fundamental Research Funds for the Central Universities, under grant 090304004, and the National Natural Science Foundation of China, under grant 60274009, and Specialized Research Fund for the Doctoral Program of Higher Education, under grant 20020145007.

\section{References}

[1] Q. Lu, S. W. Mei, T. L. Shen, et al., "Recursive Design of Nonlinear $H_{\infty}$ Excitation Controller," Science in China (SeriesE), Vol. 30, No. 1, 2000, pp. 70-78.

[2] S. J. Li, Z. Yu and G. Wang, "Adaptive H-Infinity Control of Synchronous Generators with Steam Valve via Hamiltonian Function Method," Journal of Control Theory and Applications, Vol. 4, No. 2, 2006, pp. 105-110. doi:10.1007/s11768-006-5059-6

[3] J. Ma, Z. R. Xi, S. W. Mei, et al., "Nonlinear Stabilizing Controller Design for the Steam-Valuing and Excitation System Based on Hamiltonian Energy Theory," Proceedings of the CSEE, Vol. 22, No. 5, 2002, pp. 88-93.

[4] H. R. Ri, D. H. Li and L. Q. Li, "Reheat-Type Turbo Generator Steam Valve Whole-Range Non-linear Control," Journal of Tsinghua University (Science and Technology), Vol. 40, No. 10, 2000, pp. 89-92.

[5] Y. Z. Sun, Q. Lu and C. X. Sun, "On the Study of Power System Nonlinear Robust Control," Proceedings of the CSEE, Vol. 16, No. 6, 1996, pp. 361-365.

[6] Y. Z. Sun, X. Li and H. P. Dai, "Nonlinear Excitation Controller to Improve both Power System Stability \& Voltage Regulation Accuracy," Proceedings of the CSEE, Vol. 16, No. 5, 1996, pp. 332-335.

[7] Q. Lu and Y. Z. Sun, "Nonlinear Control of Power Systems," Science Press, Beijing, 1993.

[8] B. H. Wang, C. W. Yang, Q. Zhang, et al., "Integrated Nonlinear Adaptive Backstepping Controller for Synchronous Generator," Control Theory \& Applications, Vol. 23, No. 1, 2006, pp. 60-64.

[9] Y. Y. Wang and J. David, "Robust Nonlinear Coordinated Control of Power Systems," Automatica, Vol. 32, No. 4, 1996, pp. 611-618. doi:10.1016/0005-1098(95)00 186-7

[10] Li. Y. Sun and J. Zhao, "Nonlinear Adaptive Control for the Turbine Steam Valve with Input Constraints," Control Theory \& Applications, Vol. 6, No. 6, 2009, pp. 601-606.

[11] W. L. Li, Y. W. Jing and M. S. Wang, "Stability Control in Power Systems: A Survey," Control and Decision, Vol. 17, No. 6, 2002, pp. 1-6.

[12] W. L. Li, Y. W. Jing and X. P. Liu, “Adaptive Robust Backstepping Design for Nonlinear Steam Valve Controller," Proceedings of the CSEE, Vol. 23, No. 1, 2003, pp. 155-158.

[13] W. L. Li, "Study on the Nonlinear Adaptive Robust Control for Power Systems," Ph.D. Thesis, Northeastern University, Shenyang, 2003. 